\title{
Coulisses
}

Revue de théâtre

1 | Printemps 1990

Varia

\section{Les trois batailles ou la mémoire du théâtre}

\section{Rédaction}

\section{OpenEdition}

Journals

Édition électronique

URL : http://journals.openedition.org/coulisses/1537

DOI : 10.4000/coulisses.1537

ISSN : 2546-9460

\section{Éditeur}

Presses universitaires de Franche-Comté

\section{Édition imprimée}

Date de publication : 1 avril 1990

Pagination : 3-7

ISSN : 1150-594X

\section{Référence électronique}

Rédaction, «Les trois batailles ou la mémoire du théâtre », Coulisses [En ligne], 1 | Printemps 1990, mis en ligne le 04 juillet 2017, consulté le 22 octobre 2019. URL : http://journals.openedition.org/coulisses/ 1537 ; DOI : 10.4000/coulisses. 1537

Ce document a été généré automatiquement le 22 octobre 2019

Coulisses 


\title{
Les trois batailles ou la mémoire du théâtre
}

\author{
Rédaction
}

Et si l'on veut que nous nommions plus clairement le sentiment qui nous anime, la passion qui nous pousse, nous contraint, nous oblige, à laquelle il faut que nous cédions enfin, c'est l'indignation.

Jacques Copeau

1 La phrase de Copeau qui figure en exergue résume l'action théatrale de Serge Pauthe. Cette apostrophe invitant à l'exigence éclaire le thème du spectacle donné à l'Espace Planoise en octobre dernier. Les trois batailles - celle du directeur de théâtre, de l'auteur et de l'acteur - réfèrent à trois moments forts de l'histoire du théâtre incarnés par trois hommes : Vilar, Beaumarchais, Copeau. «L'histoire de ces trois batailles, c'est de sortir de l'ombre, de populariser des aventures assez folles» (S. Pauthe). Des liens profonds existent entre ces trois itinéraires d'hommes passionnés en lutte pour la défense de leurs convictions artistiques. Pour parler de cette création, Coulisses a rencontré Serge Pauthe, l'auteur et Jean-Michel Potiron, le dramaturge qui a collaboré à l'écriture de la troisième bataille ainsi qu'à la mise en scène des deuxième et troisième parties.

\section{La bataille de Chaillot}

2 Ecrite en 1985 et jouée près de trois cent cinquante fois, c'est le volet le plus ancien du tryptique. «Il s'agit d'un spectacle qui a beaucoup changé et qui arrive à un aboutissement » (SP). À l'époque de Vilar, le théâtre en tant que patrimoine culturel était la propriété d'une fraction privilégiée de la société. Le théâtre classique appartenait ainsi à la Comédie Française et à son public. « La grande force de Vilar a été de restituer cette culture à un public qui en était privé. Le Cid joué devant des milliers de spectateurs, c'était alors quelque chose de totalement nouveau» (SP). Ce que le 
spectacle raconte, c'est l'histoire du TNP, la bataille permanente d'un directeur de théâtre - Vilar -, repérable par des dates, des faits, un ancrage dans le temps, la reconstitution d'une lutte pour une culture populaire sans concession.

\section{La bataille de Beaumarchais}

3 Cette deuxième partie date de 1988. Le pôle d'intérêt est ici la représentation de la manière dont Beaumarchais a réussi, contre la censure, à faire jouer sa pièce Le Mariage de Figaro. Je n'épouse absolument pas la cause de Beaumarchais, mais ce qui m'intéresse, c'est quand quelqu'un comme Beaumarchais fait tout au niveau de la ruse (SP). La mise en scène a été complètement revisitée pour le spectacle donné à Besançon.

4 JM Potiron :

Au départ, le Beaumarchais avait été mis en scène par Serge avec une table et un tabouret comme le Vilar. Notre exigence, c'était de faire un spectacle qui garde une cohérence pendant trois heures mais avec des parties qui aient chacune leur style, leur façon de résonner en nous. On avait envie de mettre un peu de cette joie qui ressemble à Beaumarchais. Il y a la musique, le plaisir du jeu, un esprit baroque que l'on a essayé de recréer.

\section{La bataille du Vieux Colombier}

5 Changement de ton avec le dernier volet - la bataille de l'acteur - écrit et créé à Besançon, qui met en scène la souffrance du comédien. "On a écrit, je ne sais pas, un millier de pages au moins pour aboutir à ces quarante pages et ces pages ont été un travail de douleur » (JMP). Que veut dire pour un acteur être dans les ténèbres, tel est le point d'orgue de la pièce. Dans cette bataille, plus de dates ni de faits historiques; au centre, Copeau, un homme à la recherche de la lumière pour le théâtre et les comédiens.

6 Au début du siècle, en effet, le théâtre est le lieu de la facilité et du spectaculaire. Le mélodrame - les deux orphelines, la porteuse de pain - fait recette; la comédie de boulevard, les spectacles catastrophe se multiplient : « À l'époque, le comédien était un ustensile au milieu de catastrophes, de raz de marée, de tremblements de terre. Les metteurs en scène ne considéraient plus l'acteur comme une personne qui pouvait être poétique, donner une architecture. » (JMP) Copeau, qui jusqu'à l'âge de trente-trois ans n'avait jamais fait de théâtre, s'est alors insurgé au nom de la dignité professionnelle et sociale du comédien et d'une certaine esthétique théâtrale, le « théâtre nu ».

7 La bataille du Vieux Colombier marque la clôture du spectacle de Serge Pauthe. L'ordre des batailles, qui n'est pas chronologie du temps, correspond à l'ordre d'écriture des pièces et en ce sens il est chronologie de la pensée. Serge Pauthe précise la construction de la représentation :

Si je mets Copeau à la fin, bien qu'il soit antérieur à Vilar, c'est parce qu'il est plus près de nous. Copeau, c'est quelque chose qui n'est pas fini, qui interroge toujours.

La fin du spectacle est un appel, une interpellation.

8 Ce sont donc trois moments de la mémoire du théâtre restitués au public, héritage culturel représenté sur scène où le théâtre nous parle de lui-même. 


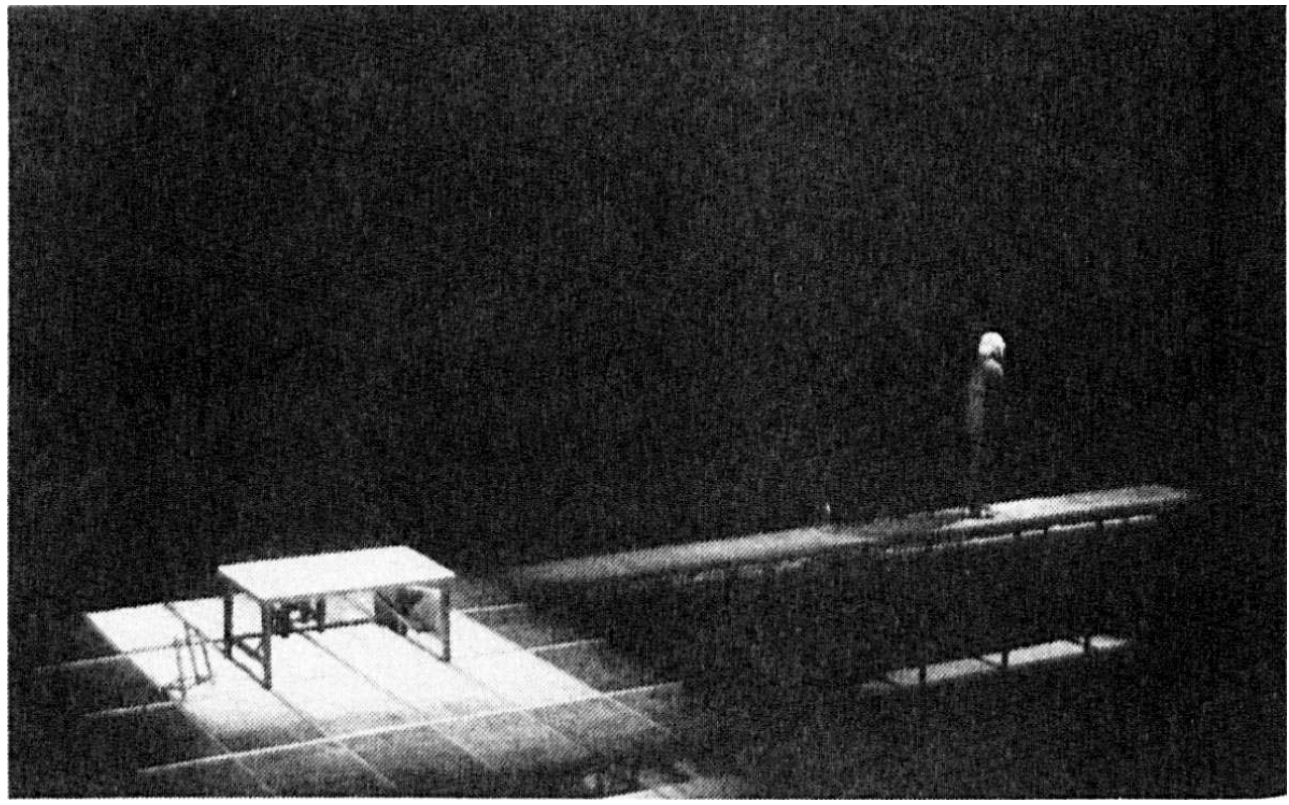

Les Trois Batailles, dispositif scénique.

(c) Valérie Goy.

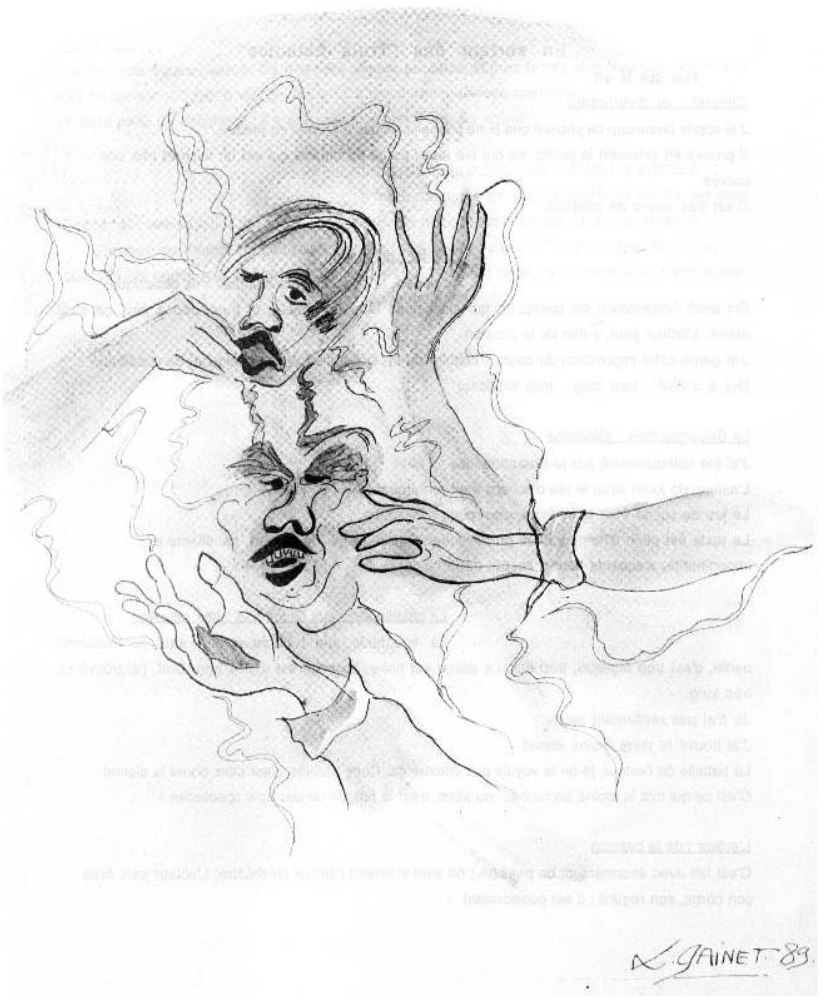

(c) Laurence Gainet, 1989.

\section{En sortant des « Trois batailles », pris sur le vif}

\section{Culturel... et divertissant}

« J'ai appris beaucoup de choses que je ne connaissais pas à propos du théâtre. » 
10 «Il prouve en amusant le public, ce qui fait aussi partie du théâtre qui est un loisir et pas une corvée."

11 «C'est très nourri de citations. »

\section{Le Vilar : didactique?}

12 «Au départ, le spectacle me semblait très didactique. »

13 « On avait l'impression de quelqu'un qui allait nous faire un cours; et puis, peu à peu, ça s'est animé. L'acteur joue, y met de la passion. »

14 «J'ai gardé cette impression du cours d'histoire du théâtre mais fait par quelqu'un de passionné Dur à suivre... trop long... trop explicatif. »

\section{Le Beaumarchais : plébiscité}

15 «J'ai été enthousiasmé par le Beaumarchais. »

16 «L'astuce de jouer avec le jeu d'échecs était remarquable. »

17 «Le jeu de scène était formidable, c'est plein d'invention. »

18 « Le texte est plein d'humour, très primesautier et la musique de Mozart, sautillante et impertinente, s'accorde bien à l'esprit de la pièce. »

\section{La bataille du Vieux Colombier : des réserves}

19 «Le spectacle m'a beaucoup plu, sauf la troisième partie, c'est trop statique, trop dur. La scène est noire. Le texte est moins bon. Bref, j'ai trouvé ça très long. »

20 «Je n'ai pas réellement suivi J’ai trouvé le texte moins abouti. »

21 «La bataille de l'acteur, je ne la voyais pas comme ça. Cette bataille, c'est sans doute la sienne."

22 «C'est ce qui m’a le moins accroché... ou alors, c'est la fatigue de ces trois spectacles!»

\section{L'acteur : de la passion...}

23 «C'est fait avec énormément de passion ; on sent vraiment l'amour du théâtre. L'acteur joue avec son corps, son regard ; il est passionnant. »

\section{... et une voix}

24 «Il a une voix bizarre, aiguë. Ca m'a paru gênant au début et puis je m’y suis habituée. En plus la voix ne correspond pas à son physique : il a une certaine densité corporelle.» «Je lui ai parlé au téléphone : il a une voix plus grave que sur scène. »

\section{La question du public : des spectateurs avertis ?}

26 "Il me semble que ce type de spectacle touche des gens relativement avertis qui s'intéressent au théâtre. Ça reste quelque chose d'assez confidentiel. » 
27 "L'histoire du théâtre ne doit pas réellement passionner le public «moyen", sélectionne un auditoire. Je pense que le Beaumarchais pourrait plaire à un large public mais il ne viendra pas, c'est ça le problème. » 